\title{
Thigmotactic responses in an open-field
}

\author{
M.R. Lamprea ${ }^{1}$, F.P. Cardenas ${ }^{2}$, J. Setem ${ }^{3}$ and S. Morato ${ }^{4}$ \\ ${ }^{1}$ Departamento de Psicología, Facultad de Ciencias Humanas, Universidad Nacional de Colombia - \\ Sede Bogotá, Bogotá, Colombia \\ Departamento de Psicología, Facultad de Ciencias Humanas, Universidad de Los Andes, Bogotá, \\ Colombia \\ ${ }^{3}$ Instituto de Estudos do Comportamento (Psicolog), Ribeirão Preto, SP, Brasil \\ ${ }^{4}$ Departamento de Psicologia e Educação, Faculdade de Filosofia, Ciências e Letras de Ribeirão Preto, \\ Universidade de São Paulo, Ribeirão Preto, SP, Brasil
}

Correspondence to: S. Morato, Departamento de Psicologia e Educação, FFCLRP, USP, Avenida Bandeirantes, 3900, 14040-901 Ribeirão Preto, SP, Brasil

Fax: +55-16-3602-4835. E-mail: smorato@ffclrp.usp.br

\begin{abstract}
The goal of the present study was to investigate the role of thigmotaxis (the tendency to remain close to vertical surfaces) in rat exploratory behavior in an open-field. Thigmotaxis was investigated in a parametric way, using 24 experimentally adult naive male Wistar rats $(210-230 \mathrm{~g})$. Exploratory behavior was studied in an open-field $(\mathrm{N}=12)$ in 5 -min sessions and behavior was analyzed in terms of where it occurred: in areas surrounded by two, one, or no walls. Another group of rats $(N=12)$ was studied in an open-field with blocks placed near two of the corners so as to make these corner areas surrounded by three walls. The floor of the open-fields was divided into 20 -cm squares in order to locate the exact place of occurrence of each behavior. The following behaviors were recorded: entries into the squares, rearings, and groomings. In both types of open-field the rats chose to remain longer in the squares surrounded by the largest possible number of walls. In one of the open-fields, the mean time (seconds) spent in squares surrounded by two walls was longer than the time spent in squares surrounded by one or no walls (37.2, 7.7, and $1.8 \mathrm{~s}$, respectively). In the other open-field, the mean time spent in squares surrounded by three walls was longer than the time spent in squares surrounded by two, one or no walls (41.7, 20.4, 7.0, and $2.6 \mathrm{~s}$, respectively). Other measures presented a similar profile. These results indicate that rats are sensitive to the number of walls in an environment and prefer to remain close to them.
\end{abstract}

Key words: Exploratory behavior; Thigmotaxis; Open-field; Rearing behavior; Grooming; Locomotion

S. Morato was the recipient of a research fellowship from CNPq (No. 351317/96-1).

Publication supported by FAPESP.

Received December 22, 2006. Accepted November 29, 2007

\section{Introduction}

The role played by different aversive stimuli in behavioral tasks that take advantage of the rat's natural tendency to explore, like the open-field or the elevated plusmaze, is not fully understood. For long it has been assumed that the innate fear of open spaces was the most important anxiety-triggering stimulus (1), an assumption that later received experimental support (e.g., 2). However, the mechanisms by which rodents perceive the open- ness of an environment are still unclear.

Barnett (1) states that "rats, like other small mammals, tend to move in contact with a vertical surface; they also eat in a corner rather than in an open space" (p. 49). In the same paragraph, he speculates that "Functionally, thigmotactic behavior is presumably an aspect of taking cover from predators". Grossen and Kelley (3) specifically narrowed this assumption to aerial predators.

In the elevated plus-maze, several aspects have been proposed as explanations for the aversion rats exhibit to 
the open arms. It has been suggested that rats are afraid of the novelty of the open arms (4). It has also been proposed that the height perceived from the open arms is the dimension causing fear and, consequently, aversion to them (5). Similarly, File (6) stated that rats previously exposed to an elevated plus-maze present fear of heights when tested again in the same apparatus. They additionally suggest that the animals acquire such a fear of heights during the initial 5-min exposure to the maze and that, given this rapid acquisition, "there may be a genetic predisposition for such a fear" (p. 494).

However, Treit et al. (2) clearly demonstrated that "open spaces, rather than height or novelty, is the anxiogenic stimulus in the elevated plus-maze" (p. 463). They also concluded that "rat's fear of the open arms is driven by thigmotaxis, a natural defensive response in which rats remain close to vertical surfaces, thereby shielding themselves from predators".

The open-field is another test that takes advantage of the rat's tendency to explore new environments, as measured by ambulation $(7,8)$. Typically, rats are more active and remain longer in the periphery of the apparatus in comparison to the central areas, mainly in the first session, a preference that has been interpreted as being determined by thigmotaxis (9).

Since, to our knowledge, there are no experiments investigating the role of thigmotaxis per se (i.e., thigmotaxis is usually a given characteristic of the apparatus), the

\begin{tabular}{|c|c|c|c|c|c|}
\hline 3 & 1 & 1 & 1 & 1 & 2 \\
\hline 3 & 1 & 0 & 0 & 0 & 1 \\
\hline 2 & 0 & 0 & 0 & 0 & 1 \\
\hline 1 & 0 & 0 & 0 & 0 & 2 \\
\hline 2 & 1 & 1 & 1 & 1 & 3 \\
\hline
\end{tabular}

Figure 1. Diagram of the open-field. Each line is $20 \mathrm{~cm}$ long and the digit inside the divisions indicates the number of walls surrounding it. $B$ indicates the location where the blocks were placed. For details, see text. goal of the present study was to investigate the role of vertical surfaces in a more parametric way. This was carried out by testing rats in a modified open-field that had divisions with different numbers of walls.

\section{Material and Methods}

\section{Subjects}

Adult male Wistar-derived rats weighing 213-227 g obtained from the vivarium of the University of São Paulo at Ribeirão Preto, with free access to food and water were housed 6 to a cage $(40 \times 33 \times 16 \mathrm{~cm})$, on a 12-h dark-light cycle (lights on at 7:00 am). All behavioral tests occurred in the light phase of the cycle, before 11:30 am.

\section{Apparatus}

A 1.2-m square open-field with a dark brown Formica floor surrounded by $50-\mathrm{cm}$ high wooden walls was used. The tests were carried out in a room lit by a 60 -W light bulb $1.75 \mathrm{~m}$ above the center of the open-field (150 lux at the center of the apparatus). The experimental sessions were recorded with a video camera interfaced with a television set and a video cassette recorder in an adjacent room. One group of rats $(\mathrm{N}=12)$ was tested in a standard way, with the open-field divided into three areas according to the number of adjacent walls: a) two walls (corners, luminosity: 147 lux), b) one wall (the laterals, 147 lux), and c) no walls (center, 150 lux). The maximum number of walls possible in a square of the apparatus was two and the apparatus was designated $2 \mathrm{~W}$ open-field. Another group of animals $(N=12)$ was tested in the open-field with two $20 \times 20 \times 30$ $\mathrm{cm}$ blocks placed inside and near two of the corners so as to form, in addition to the areas described in (a), (b), and (c), two $20 \times 20 \mathrm{~cm}$ areas with three walls each (luminosity: 146 lux; see Figure 1). Thus, the maximum possible number of walls in this apparatus was three and the apparatus was designated $3 \mathrm{~W}$.

\section{Procedure}

Each rat was placed in the center of the open-field and allowed to freely explore for $5 \mathrm{~min}$. After each test, the open-field was cleaned with $10 \%$ ethanol, and then dried with a dry cloth. In order to record locomotor activity online, the image of the open-field on the television screen was divided into thirty-six $20 \times 20 \mathrm{~cm}$ squares. Every time both hind paws entered one of these squares, a crossing was recorded. The total distance run by the animals was estimated from the number of crossings. Rearing and grooming frequencies and the time spent rearing and grooming were also recorded and analyzed in terms of the area of occurrence. A rearing episode was recorded when- 
ever the rat stood on its hind paws with the body at an angle with the floor greater than $45^{\circ}$. A grooming episode was defined by repetitive movements of front paws or mouth on the fur. We were also prepared to record stretching behavior but none occurred.

\section{Statistical analysis}

When absolute measures were not used, a mean transformation was employed, obtained by adding the measures recorded for single squares of one type (e.g., 3W) and dividing them by the total number of squares of that type. One-way analysis of variance (ANOVA) for repeated measures was used to detect differences between data referring to areas with different numbers of surrounding walls. When ANOVA was significant, the Tukey test for multiple comparisons between means was performed. Differences between the two arrangements of the open-field were determined by the Student $t$-test. In all cases, the level of statistical significance was set to $P<0.05$.

\section{Results}

Animals tested in the $2 \mathrm{~W}$ and $3 \mathrm{~W}$ open-fields exhibited a very similar level of locomotor activity during the 5-min session when the total number of crossings was analyzed. The $t$-test $(t(22)=-0.155, \mathrm{P}=0.878)$ showed no differences in the distance run by $2 \mathrm{~W}(24.4 \pm 1.91 \mathrm{~m})$ and $3 \mathrm{~W}$ rats $(24.8 \pm 1.40 \mathrm{~m})$. Considering the different areas of the $2 \mathrm{~W}$ and $3 \mathrm{~W}$ open-fields, the animals avoided entering in the central area and spent little time there but they did run along the walls, i.e., squares with one wall, without remaining much time in them (Figure 2). The rats in the $3 \mathrm{~W}$ openfield entered the non-walled squares significantly more but did not differ in terms of time spent in these squares. The animals in the $2 \mathrm{~W}$ open-field remained longer in the twowalled squares than the ones in the $3 \mathrm{~W}$ open-field, but these seemed to distribute their time in both two- and three-walled squares.

The $t$-test showed no differences between $2 \mathrm{~W}$ and $3 \mathrm{~W}$ rats in the total number of rearings $(24.58 \pm 2.06$ and 28.33 \pm 2.19 , respectively; $t(22)=-1.247, P=0.225)$, total rearing time $(29.47 \pm 3.29$ and $39.75 \pm 3.97 \mathrm{~s} ; t(22)=-1.998, \mathrm{P}=$ $0.058)$ and mean rearing time (1.2 \pm 0.10 and $1.40 \pm 0.07$ $\mathrm{s} ; t(22)=-1.662, \mathrm{P}=0.111)$. When comparing the frequency and rearing time in the squares, again there were no differences between subjects tested in the $2 \mathrm{~W}$ and $3 \mathrm{~W}$ open-fields. Both groups, however, exhibited more of this behavior in squares surrounded by more walls (Figure 3 ).

The $t$-test $(t(22)=-2.358, \mathrm{P}=0.028)$ showed that the rats in the $3 \mathrm{~W}$ open-field groomed more often (13.08 \pm $2.12)$ than those in the $2 \mathrm{~W}$ open-field $(7.75 \pm 0.79)$ but did not differ in the time they spent grooming (3W: $29.44 \pm 4.68$ $\mathrm{s} ; 2 \mathrm{~W}: 29.52 \pm 4.40 \mathrm{~s} ; t(22)=0.013, \mathrm{P}=0.990)$, which, in turn, altered the grooming pattern in the sense that the mean grooming episode lasted less in $3 \mathrm{~W}$ than in $2 \mathrm{~W}$ subjects (3W: $2.51 \pm 0.41 \mathrm{~s} ; 2 \mathrm{~W}: 4.23 \pm 0.64 \mathrm{~s} ; t(22)=$ $2.261, P=0.034)$. Figure 4 shows grooming in the different squares. There were no differences between the animals in the two open-fields but the animals groomed more in squares surrounded by more walls. The animals in the $2 \mathrm{~W}$ open-field groomed more in the two-walled squares than the animals in the $3 \mathrm{~W}$ open-field but, as happened with the entries, these seemed to distribute their time in both twoand three-walled squares

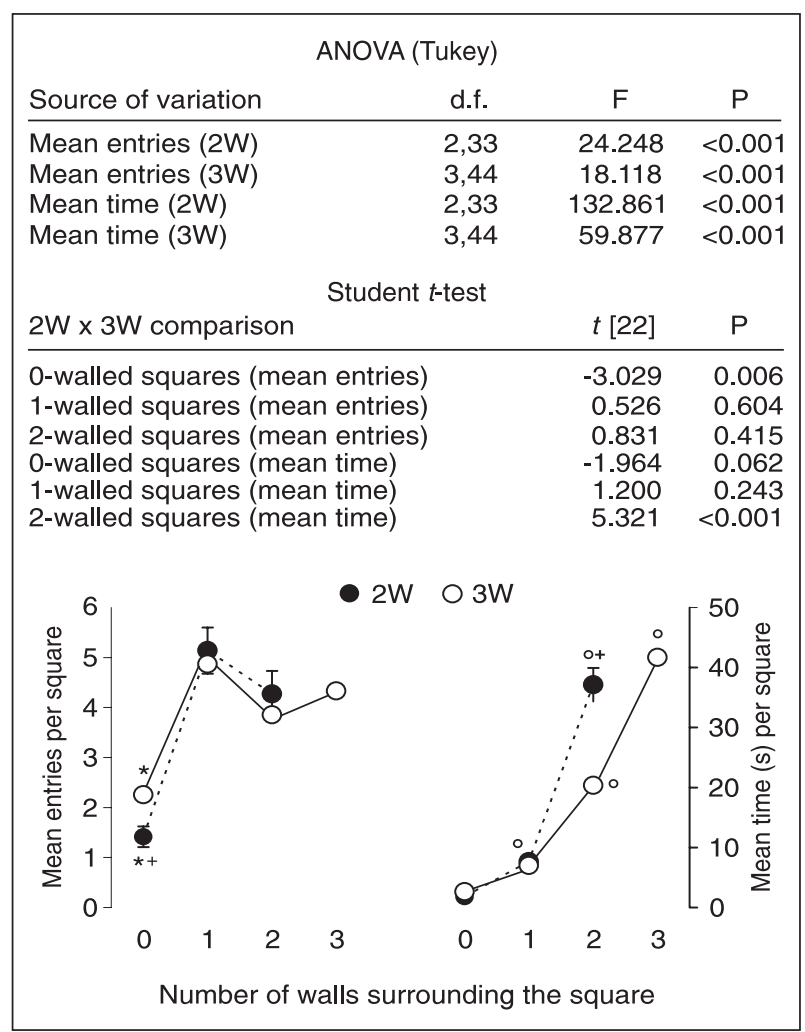

Figure 2. Mean frequency of entries and mean time spent per square. These values were obtained by dividing the totals occurring in squares with a certain number of walls surrounding them and dividing by the number of walls. $2 \mathrm{~W}=$ open-field in which the maximum number of walls per square was two or $3 \mathrm{~W}=$ openfield with the block that allowed a maximum number of three walls in certain squares. d.f. $=$ degrees of freedom; $\mathrm{F}=$ statistical F value; $t=$ statistical $t$ value for the specified number of d.f.; $\mathrm{P}=$ probability. ${ }^{*} \mathrm{P}<0.05$ compared to squares with a larger number of walls; ${ }^{\circ} \mathrm{P}<0.05$ compared to squares with a smaller number of walls (ANOVA followed by the Tukey test). ${ }^{+} \mathrm{P}<0.05$ compared to different $3 \mathrm{~W}$ (Student $t$-test). 


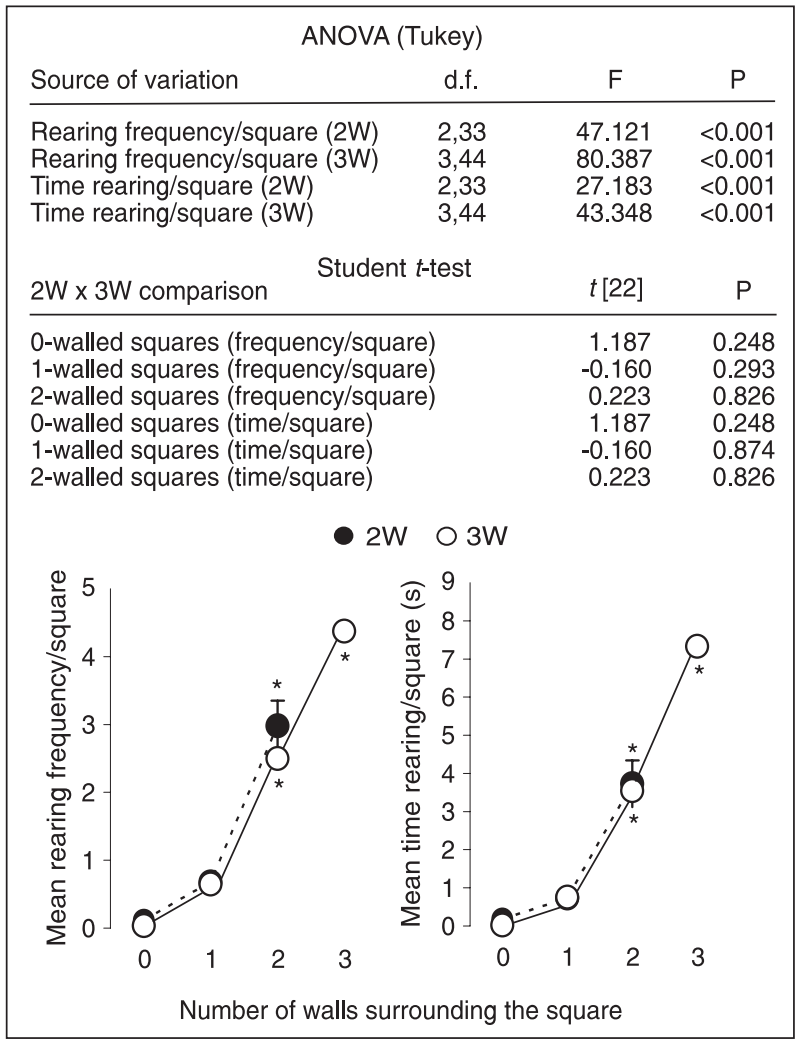

Figure 3. Mean frequency of rearings and mean time rearing per square. These values were obtained by dividing the totals occurring in squares with a certain number of walls surrounding them and dividing by the number of walls. $2 \mathrm{~W}=$ open-field in which the maximum number of walls per square was two or $3 \mathrm{~W}=$ openfield with the block that allowed a maximum number of three walls in certain squares. d.f. $=$ degrees of freedom; $F=$ statistical $\mathrm{F}$ value; $t=$ statistical $t$ value for the specified number of d.f.; $\mathrm{P}=$ probability. ${ }^{*} \mathrm{P}<0.05$ compared to squares with a smaller number of walls (ANOVA followed by the Tukey test).

\section{Discussion}

The rats in both open-fields exhibited no differences in locomotor activity, as shown by the almost identical distances run in both apparatuses during the sessions. The number of squares entered shows that the rats preferred to enter squares with at least one adjacent wall, indicated by the lower number of entries into squares with no walls and a similar number of entries into squares with one, two or three adjacent walls. However, the time the rats remained in each square was a function of the number of walls. When squares with three adjacent walls were available to the animals they preferred to remain in them at the expense of the time allocated to squares with two adjacent walls, but

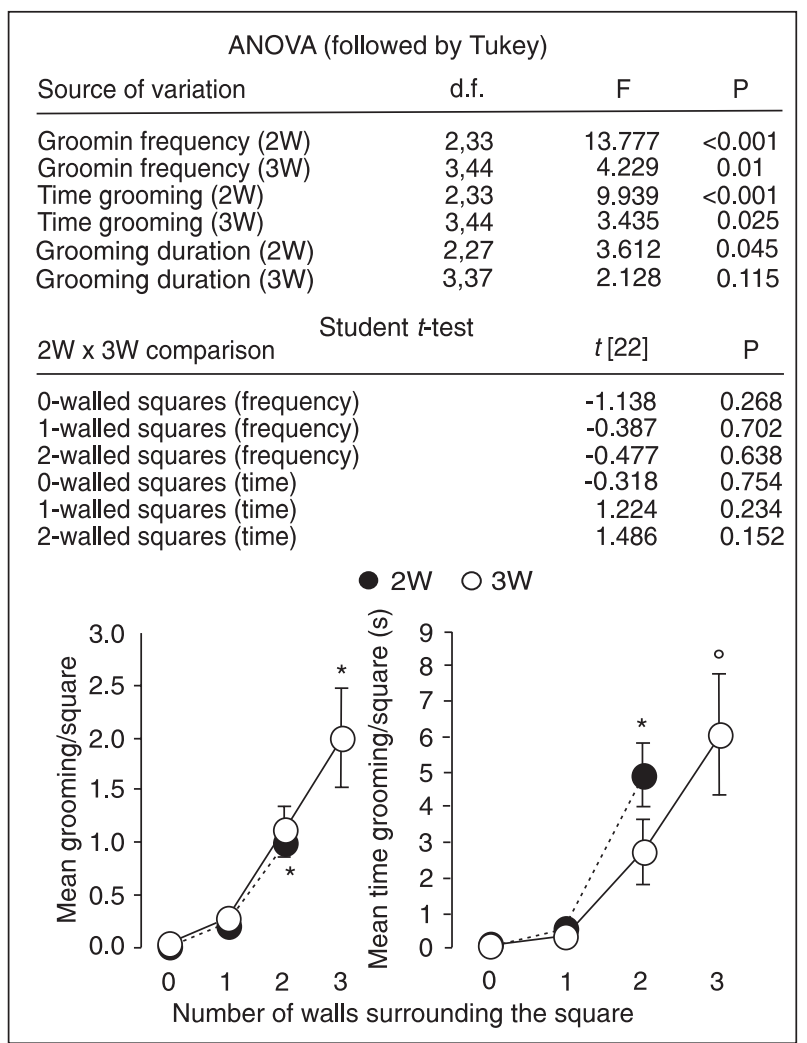

Figure 4. Mean frequency of groomings and mean time grooming per square. These values were obtained dividing the totals occurring in squares with a certain number of walls surrounding them and dividing by the number of walls. $2 \mathrm{~W}=$ open-field in which the maximum amount of walls per square is two or $3 \mathrm{~W}=$ open-field with the block that allowed a maximum number of three walls in certain squares. d.f. $=$ degrees of freedom; $F=$ statistical $\mathrm{F}$ value; $t=$ statistical $t$ value for the specified number of d.f.; $P=$ probability. ${ }^{*} P<0.05$ compared to squares with a smaller number of walls; ${ }^{P} P<0.05$ compared to squares with no walls and one wall (ANOVA followed by the Tukey test).

not at the expense of squares with no or just one adjacent wall. In addition, the rats spent an amount of time in the squares with two adjacent walls in the $2 \mathrm{~W}$ open-field similar to the amount of time spent in the squares with three adjacent walls in the $3 \mathrm{~W}$ open-field. This indicates that, at least for this kind of exploratory behavior, the rats use thigmotaxis in order to choose where and how long to remain in the different areas of the apparatus. It also indicates that the rats preferred to remain in the most secure area of the open-field, since the occupation of the two-walled squares in the $2 \mathrm{~W}$ open-field was similar to that of the three-walled squares in the $3 \mathrm{~W}$ open-field.

As far as thigmotaxis is concerned, it is interesting to notice that in both apparatuses the rats avoided the central 
area, composed of squares with no adjacent walls. As incursions in the central area are correlated with a decrease in anxiety (10), the presence of three-walled squares did not affect whatever anxiety the apparatuses may have caused. In this respect, it may be argued that the rats preferred squares with the largest possible number of adjacent walls because, by allowing more thigmotaxis, these squares elicited less anxiety. Also, this is similar to what happens in a widely used anxiety model, the elevated plus-maze: rats usually prefer to remain in the arms surrounded by walls and avoid the open arm, probably for the same motivational reasons as the central area of an openfield $(10,11)$. Additionally, Horev et al. (12) showed a similar effect in the trajectory of the mouse in an open-field. They suggested a double-influence of the wall on the mouse's exploratory behavior: one of guidance and the other of attraction. The first function is suggested because the animals progress in parallel to the wall guiding the heading direction and increasing the speed of locomotion when they are more parallel to the wall. The attraction influence is reflected by the fact that mice tend to perform a large component of their activity near the wall, including most of their progression, considering this to be one aspect of thigmotaxis.

Another behavior considered to be of an exploratory nature is rearing (13-15). Like the exploratory behavior already discussed, the frequency of rearing did not differ in any important way between the $2 \mathrm{~W}$ and $3 \mathrm{~W}$ open-fields. Also similar was the time allocated to rearing according to the number of adjacent walls, except that the more the adjacent walls, the more time the rats reared in a square. No or almost no rearings occurred in the central area of the two open-fields, perhaps because the walls offered more stimuli to be explored by rearing. It remains to be determined what relationship, if any, exists between rearing behavior and thigmotaxis.

Unlike the measures discussed so far, grooming was sensitive to the differences between the $2 \mathrm{~W}$ and $3 \mathrm{~W}$ openfields: the rats groomed more often in the $3 \mathrm{~W}$ open-field but the total time used in this activity was the same in both apparatuses. This shows that there was a difference in the temporal pattern of grooming between groups, i.e., rats in the $3 \mathrm{~W}$ open-field groomed more frequently but each episode lasted less time than in the $2 \mathrm{~W}$ open-field. In

\section{References}

1. Barnett SA. The rat: $A$ study in behavior. Chicago: The University of Chicago Press; 1975.

2. Treit D, Menard J, Royan C. Anxiogenic stimuli in the elevated plus-maze. Pharmacol Biochem Behav 1993; 44: addition, the rats spent similar total amounts of time in both apparatuses, but allocated more time to the squares with the higher number of adjacent walls, either two or three, according to the open-field they were tested in. A similar time allocation pattern was observed for exploratory behavior. It is difficult to attribute motivational properties to this behavior since in factor analysis studies grooming loads similarly on the different (and perhaps incompatible) factors of exploration and anxiety $(13,15)$. Our data, however, do support an exploratory nature of grooming rather than an eventual connection with anxiety, since the mean grooming frequency exhibited a similar profile as the mean rearing frequency while the mean time grooming exhibited a similar profile as the mean time spent in each square and both measures correlated well with exploration.

Our results do not allow us to identify the factor guiding the rats choice of squares with as many walls as possible. One explanation could be that the rats just see the walls and choose to remain in squares with more walls surrounding them. This is supported by the data reported by Martínez et al. (16) suggesting that the exploratory behavior of rats in the elevated plus-maze is dependent on vision. An alternative explanation could be that rats are using their vibrissae to recognize the squares with more walls. However, Milani et al. (17) showed that rats with the vibrissae of one side of the head removed do not increase the total time they are in contact with the wall of a circular open-field, thus indicating that the animals are not using the vibrissae for this task.

In general, the data reported in the present experiment show that exploratory and other behaviors tend to occur more in squares surrounded by walls. We do not think this behavioral pattern was influenced by the amount of light reaching each square since, on the one hand, one- and two-walled squares were used differently by the rats in spite of receiving the same amount of light, and on the other hand, the difference between the amount of light in two- and three-walled squares was too little to matter, indicating that the animals were sensitive to the number of walls. The larger the number of walls, the more behavior occurs in a particular square. This indicates that thigmotaxis is a determinant factor of rat behavior in unfamiliar places and that the rats are sensitive to the number of walls near which they can choose to be.
463-469.

3. Grossen NE, Kelley MJ. Species-specific behavior and acquisition of avoidance behavior in rats. J Comp Physiol Psychol 1972; 81: 307-310. 
4. Montgomery KC. The relation between fear induced by novel stimulation and exploratory behavior. J Comp Physiol Psychol 1955; 48: 254-260.

5. Pellow S, File SE. Anxiolytic and anxiogenic drug effects on exploratory activity in an elevated plus-maze: a novel test of anxiety in the rat. Pharmacol Biochem Behav 1986; 24: 525529.

6. File SE. The interplay of learning and anxiety in the elevated plus-maze. Behav Brain Res 1993; 58: 199-202.

7. Walsh RN, Cummins RA. The Open-Field Test: a critical review. Psychol Bull 1976; 83: 482-504.

8. Nahas TR. O teste do campo aberto. In: Xavier GF (Editor), Técnicas para o estudo do sistema nervoso. São Paulo: Editora Plêiade; 1999.

9. Valle FP. Effects of strain, sex, and illumination on openfield behavior of rats. Am J Psychol 1970; 83: 103-111.

10. Lister RG. Ethologically-based animal models of anxiety disorders. Pharmacol Ther 1990; 46: 321-340.

11. Carobrez AP, Bertoglio LJ. Ethological and temporal analyses of anxiety-like behavior: the elevated plus-maze model
20 years on. Neurosci Biobehav Rev 2005; 29: 1193-1205.

12. Horev G, Benjamini Y, Sakov A, Golani I. Estimating wall guidance and attraction in mouse free locomotor behavior. Genes Brain Behav 2007; 6: 30-41.

13. Cruz AP, Frei F, Graeff FG. Ethopharmacological analysis of rat behavior on the elevated plus-maze. Pharmacol Biochem Behav 1994; 49: 171-176.

14. Hogg S. A review of the validity and variability of the elevated plus-maze as an animal model of anxiety. Pharmacol Biochem Behav 1996; 54: 21-30.

15. Anseloni VZ, Brandão ML. Ethopharmacological analysis of behaviour of rats using variations of the elevated plusmaze. Behav Pharmacol 1997; 8: 533-540.

16. Martínez JC, Cardenas F, Lamprea M, Morato S. The role of vision and proprioception in the aversion of rats to the open arms of an elevated plus-maze. Behav Processes 2002; 60: 15-26.

17. Milani H, Steiner H, Huston JP. Analysis of recovery from behavioral asymmetries induced by unilateral removal of vibrissae in the rat. Behav Neurosci 1989; 103: 1067-1074. 